\title{
Cost-effectiveness of a health-social partnership transitional program for post-discharge medical patients
}

Frances Kam Yuet Wong ${ }^{1 *+}$, June Chau ${ }^{2+}$, Ching So ${ }^{2+}$, Stanley Ku Fu Tam ${ }^{3+}$ and Sarah McGhee ${ }^{2+}$

\begin{abstract}
Background: Readmissions are costly and have implications for quality of care. Studies have been reported to support effects of transitional care programs in reducing hospital readmissions and enhancing clinical outcomes. However, there is a paucity of studies executing full economic evaluation to assess the cost-effectiveness of these transitional care programs. This study is therefore launched to fill this knowledge gap.

Methods: Cost-effectiveness analysis was conducted alongside a randomized controlled trial that examined the effects of a Health-Social Transitional Care Management Program (HSTCMP) for medical patients discharged from an acute regional hospital in Hong Kong. The cost and health outcomes were compared between the patients receiving the HSTCMP and usual care. The total costs comprised the pre-program, program, and healthcare utilization costs. Quality of life was measured with SF-36 and transformed to utility values between 0 and 1.

Results: The readmission rates within 28 (control 10.2\%, study 4.0\%) and 84 days (control 19.4\%, study 8.1\%) were significantly higher in the control group. Utility values showed no difference between the control and study groups at baseline $(p=0.308)$. Utility values for the study group were significantly higher than in the control group at 28 $(p<0.001)$ and 84 days $(p=0.002)$. The study group also had a significantly higher QALYs gain $(p<0.001)$ over time at 28 and 84 days when compared with the control group. The intervention had an $89 \%$ chance of being cost-effective at the threshold of $£ 20000 /$ QALY.

Conclusions: Previous studies on transitional care focused mainly on clinical outcomes and not too many included cost as an outcome measure. Studies examining the cost-effectiveness of the post-discharge support services are scanty. This study is the first to examine the cost-effectiveness of a transitional care program that used nurse-led services participated by volunteers. Results have shown that a health-social partnership transitional care program is cost-effective in reducing healthcare costs and attaining QALY gains. Economic evaluation helps to inform funders and guide decisions for the effective use of competing healthcare resources.
\end{abstract}

Keywords: Health-social transitional care, Readmission, Cost-effective analysis

\section{Background}

Patients who are discharged from the hospital and readmitted within a short time are a considerable cause for concern among healthcare providers. Rehospitalizations place a financial burden on hospitals particularly those using public funding. Multiple hospital admissions also tend to compromise patients' quality of life. Many

\footnotetext{
* Correspondence: frances.wong@polyu.edu.hk

${ }^{\dagger}$ Equal contributors

'School of Nursing, The Hong Kong Polytechnic University, Hunghom, Kowloon, Hong Kong, China SAR

Full list of author information is available at the end of the article
}

transitional care programs have been implemented to support patients after hospital discharge, and the effectiveness of these programs has been examined. Studies that produced positive results in controlling hospital readmissions and enhancing quality of life tend to share some common features. These more successful programs are in general comprehensive and well supported with a multi-disciplinary care team [1-3]. The nurse usually plays a pivotal role in the team, including in the provision of direct home-based or telephone follow-up care $[4,5]$ and indirect care such as coordination. Care coordination

\section{Biomed Central}

(c) 2012 Wong et al.; licensee BioMed Central Ltd. This is an Open Access article distributed under the terms of the Creative Commons Attribution License (http://creativecommons.org/licenses/by/2.0), which permits unrestricted use, distribution, and reproduction in any medium, provided the original work is properly cited. 
involves negotiating care with multiple providers, making referrals and ensuring that the program is delivered in compliance with the set protocol $[4,6]$. Patient education is an essential element of these transitional care programs that are targeted at reinforcing health behavior, empowering self-care, enhancing treatment adherence, and monitoring signs and symptoms [5,7]. The intervention provided is proactive, continuous and regular $[2,8]$. Wong et al. [6] summarized the key features of the transitional care described above with 4Cs, which are comprehensiveness, continuity, coordination and collaboration.

The current literature has provided substantial evidence to support the thesis that transitional care programs can produce positive outcomes in regard to the reduction of readmission rates and the enhancement of quality of life and care satisfaction $[4,6,9]$. However, cost is not usually included as one of the outcome measures. When cost is included, the study often simply reports the expenditure and possible cost savings in a descriptive manner. Ledgwidge et al. [10] provided multidisciplinary care for heart failure patients for 3 months, and the cost of the service hospitalization prevented was estimated to be $€ 586$. Another study using geriatricians to target medication review, self-disease management and communication among health professionals achieved a cost saving of $€ 519$ per participant for a 6-month follow-up [11]. Naylor et al. [4] used advanced practice nurses to provide home follow-up for elderly patients for 3 months, finding significantly lower healthcare costs for the intervention group and yielding estimated mean cost savings of US $\$ 4,845$ per patient. Kwok et al. [12] also used nurse home visits provided to elderly patients for 6 months and reported a marginal difference $(p=0.048)$ of healthcare costs between the intervention and control group, with a median difference of US\$2024. Miller et al. [13] introduced early discharge supported by home visits and rehabilitation service for older people, and the cost for the intervention group was $£ 1727 \quad(\mathrm{p}=0.054)$ lower than that for the usual group. Wong et al. [2] similarly used nurse specialist follow-up for early discharged diabetic patients and was able to reduce the length of stay by 3.7 days $(\mathrm{p}<.001)$ and saved US\$1633 per patient.

There is a paucity of studies executing full economic evaluation, which considers both cost and outcomes [14], for transitional care programs. Amongst the few studies that have been published, Patel et al. [15] conducted an analysis using the outcome measures of cost and qualityadjusted life year (QALY) gain when comparing the effects of domiciliary care for stroke patients with stroke unit or stroke team care. Similar QALY gains were found among the three groups, but there was a 59\% probability that domiciliary care was cost-effective. The mean healthcare and social care costs for stroke unit, stroke team and domiciliary care were respectively £11450, £9527, and
$£ 6840$ for over 12 months. Stewart et al. [16] performed an economic modeling for estimating the cost savings achieved by three different types of UK-wide programs for patient follow-up using specialist nurse management. The analysis was based on existing and projected healthcare data and cost. The calculated expenses for home-, clinic- and home- plus clinic-based follow-up were $£ 69.4, £ 73.1$ and $£ 72.5$ million per annum respectively. The programs were estimated to be able to bring about $38.5 \%$ (home-based), 40.6\% (clinic-based) and 40.3\% (home plus clinic-based) reduction in recurrent bed utilization. It was projected that a home-based program using specialist nurse management could bring about annual savings equivalent to $£ 169000$ per 1000 patients treated.

Based on the available literature, it is uncertain whether transitional care programs especially those adopting a health-social partnership approach, are costeffective in supporting patients returning to the community after hospitalization. Many of the studies focused on clinical outcomes only. This study is therefore launched to fill this knowledge gap by examining the cost-effectiveness of a health-social partnership transitional care program for patients discharged from hospitals.

\section{Methods}

\section{Ethics}

The study protocols were reviewed and ethical approval was obtained from the Research Ethics Committee of the study hospital (Reference KC/KE-08-0990/ER-5) and the Human Subjects Ethics Sub-committee at the university with which the principal investigator was affiliated (Reference HSEAR20050920001). All potential subjects have received full explanation of the study and the agreed participants had to sign a consent form. The confidentiality and anonymity of the participants were protected and all data were identified by case numbers only.

\section{Randomized controlled trial}

This study was conducted alongside a randomized controlled trial. The details of the study have been described elsewhere [6]. Briefly, it involved medical patients discharged from an acute regional hospital in Hong Kong who met specific inclusion criteria, namely (a) being aged 60 or above, (b) MMSE $>20$, (c) ability to speak Cantonese, (d) living within the hospital service area, and (e) ability to be contacted by phone. The exclusion criteria were (a) having been discharged from institutional care, (b) being followed up by designated disease management programs, (c) inability to communicate, and (d) dying. The subjects who consented to participate were randomized into control and intervention groups. The control group received usual discharge care and the intervention group received both usual care and a 
health-social partnership transitional care management program (HSTCMP) at discharge.

The HSTCMP was a 4-week program with weekly planned events delivered by the nurse case manager (NCM) and trained volunteers (TV), guided by protocols and structured documentations. Both the NCM and TVs have received training to equip them to provide the interventions. In week 1, the NCM and the TV would conduct a home visit together. The NCM would conduct an assessment in the domains of environment, physical, psychosocial and health-related behaviors and provide relevant intervention based on the Omaha System. The Omaha System is originally developed in the United States and used widely in community care [17]. The research team has used the Omaha System in the local community and found it comprehensive and valid for use in the local context [6]. In supporting the NCM, the TVs would provide social support to the patient mainly in the emotional dimensions by expressing concerns and showing support [18]. The TVs had a resource kit on healthy home environment and available community resources if they need to provide some relevant information to the patients. At the end of the visit, the NCM would set mutual goals with the patient to enhance health. In week 2, the NCM would make a follow-up telephone call based on the Omaha System and review the mutual health goals set in week 1 . In week 3 , the TVs would conduct a home visit in pairs. The social visit was to show emotional support to the patient and see if there were needs for referrals to the social workers for further social assessment and interventions such as daily living assistance, housing assistance, and counseling. In week 4, the NCM would make the final telephone call to the client. The NCM would assess the client's health needs, monitor progress, provide health advice, reinforce health self-management behavior, assess the need for referral, and review the health goals with the patients. The NCM assumes the overall responsibility in the transitional program, and is supported by the TVs and social worker in the social aspects. The health-social care team and the researchers held regular case reviews to ensure that the intervention was delivered according to the set protocols and to discuss issues of concerns.

\section{Cost and health outcomes}

The cost and health outcomes were compared between the patients receiving the HSTCMP and usual care. The economic evaluation only involved direct costs from healthcare providers and patients. Indirect costs such as productivity loss were not considered to be relevant, since most patients were either retired (94\%) or not in full-time employment (4\%). Data on health services utilization, including the number of readmissions and length of stay of each admission, were extracted from the hospital information systems. Quality of life (QOL) was measured with the Hong Kong Chinese version of the 36-item Short-Form Health Survey (SF-36 HK). Using a locally developed algorithm [19], we transformed the health states described by the SF-36 HK into utility values between 0 and 1 . These utility values could be used to calculate the quality-adjusted life years (QALYs) when multiplied by the length of time spent in that health state. For patients who missed either one of the follow-up visits, the missing QOL values were imputed using a regression equation derived from all patients with complete QOL data, adjusted for age, gender and treatment group. Other missing follow-up data were replaced with the group means. The average QALYs gained in 28- and 84-day periods were estimated based on the change from baseline utility over the relevant period. Net monetary benefit, number of QALYs gained from the intervention and costs per QALY gained were calculated. The costing was based on the most current prices available and took a societal perspective. Total costs in the study group included the pre-program cost, program cost, and healthcare cost due to readmission and associated accident and emergency room attendance, while for the control group, only healthcare cost was included.

\section{Pre-program cost}

The pre-program cost included the training time spent by the volunteers and staff in preparation for the delivery of the intervention. The unit cost of training for volunteers and staff was estimated by multiplying the duration of training with the hourly pay based on the corresponding salary. The total costs were estimated by multiplying the unit cost for the training with the number of volunteers trained or number of staff needed for the training. The monetary values for volunteers were estimated referencing the median salary and weekly hours of work from the Census and Statistics Department [20]. As for the staff, the appropriate hourly salary levels from the Master Pay Scale [21] were used for computation.

\section{Program cost}

The program costs included the estimation of patient and staff time spent during intervention following the standard protocol. The unit costs of the program for patient and staff were estimated by multiplying the total time of intervention for each case with the hourly pay based on the corresponding salary. The total costs were estimated by multiplying the unit cost by the total number of cases in the intervention program.

\section{Healthcare cost}

The healthcare cost included all direct medical costs due to readmission, which were calculated by multiplying the 
unit cost of HK\$3650 (US\$1 = HK\$7.75) per hospital day with the length of stay for the readmission. Only readmissions related to index admission were counted. An addition of HK\$820 [22] was added if the patient was admitted through the Accident and Emergency department. The total healthcare cost was therefore taken as the sum of the total bed day costs and the total cost of emergency admissions.

\section{Cost-effectiveness analysis}

The bootstrap method was used to estimate confidence intervals for the difference in health outcome and cost between the two groups. It is an appropriate method of choice when the sampling distribution is unknown [23], and this is true in our case where the difference in health outcome and cost between groups is not certain. To estimate the cost-effectiveness of the intervention, the cost and QALYs gained at 28 and 84 days were estimated for both groups. Incremental cost-effectiveness ratios (ICER) between the groups were calculated by dividing the difference in cost by the difference in QALYs.

\section{Sensitivity analyses}

Uncertainties around the parameters were tested with one-way and probabilistic sensitivity analyses. In the one-way sensitivity analysis, intervention cost and readmission rate were tested with a $30 \%$ variation to the base value for each item. For the readmission cost, we varied the length of stay (LOS) of re-admission episodes with a minimum and maximum value of the $95 \%$ confidence intervals of the LOS for the study and control groups separately. The overall LOS of re-admission episodes from all patients was also tested to assess the impact when the difference between the groups was ignored. In the probabilistic sensitivity analysis, random values for all parameters were selected from appropriate distributions for each parameter. The pre-program and program cost and readmission rate were varied within the range of $\pm 30 \%$ of the base values using a uniform distribution. The LOS and utility scores were varied by fitting uniform distributions within the $95 \% \mathrm{CI}$ of the values obtained in the study. ICERs were generated 1000 times with a random value for each parameter every time. The results were plotted on a cost-effectiveness plane and displayed with cost-effectiveness acceptability curves. All analyses were carried out using Microsoft Excel 2007 and STATA 10.1.

\section{Results}

Among the 555 patients recruited, 283 received usual discharge care and 272 received both usual care and the health-social partnership transitional care management program. The readmission rates within 28 (control 10.2\%, study $4.0 \%$ ) and 84 days (control $19.4 \%$, study $8.1 \%$ ) were significantly higher in the control group. There was no significant difference in the LOS at readmission per patient between groups at 28 and 84 days. Utility values showed no difference between the control and study groups at baseline $(\mathrm{p}=0.308)$. There was a significant within-group difference for both the study group ( $\mathrm{p}<0.001)$ and the control group ( $p=0.011)$ over time. However, when compared between groups, utility was significantly higher for the study group than the control group at $28(\mathrm{p}<0.001)$ and 84 days $(\mathrm{p}=0.002)$. The study group also had a significantly higher QALYs gain $(\mathrm{p}<0.001)$ over time at 28 and 84 days when compared with the control group (Table 1).

The intervention cost, which included the preprogram training, staff and patient costs, was HK\$1225 per subject for the study group (Table 2). The cost of readmission per subject within 28 and 84 days was lower in the study group than in the control group, and the differences were -HK\$1505 (95\% CI: $-\$ 2670,-\$ 555)$ and $-\mathrm{HK} \$ 3000(-\$ 5104,-\$ 1211)$ for the two time periods respectively.

The intervention resulted in cost savings at both 28 and 84 days, and there were gains in QALYs of 0.002 and 0.009 respectively (Table 3 ). One-way sensitivity analyses showed that raising the intervention cost or reducing the readmission rate and length of stay for both groups by $30 \%$ would increase the ICERs at 28 days, by up to HK\$200,000 per QALY, whereas the ICERs at 84 days remained cost saving in all one-way sensitivity analyses.

The cost-effectiveness plane in Figure 1 shows that the intervention has a $65 \%$ and $95 \%$ chance of being cost saving at 28 and 84 days respectively. Cost-effectiveness acceptability curves in Figure 2 show that the intervention has an $89 \%$ chance of being cost-effective at the NICE threshold of $£ 20000$ (HK\$240000, $£ 1=H K \$ 12)$ [24].

\section{Discussion}

This paper is original and contributes to the literature by providing evidence to show that a health-social partnership transitional care program is cost-effective in reducing healthcare costs and attaining QALY gains. Previous studies have provided evidence to support that transitional care can help reduce hospital readmissions and enhance clinical outcomes. However, these interventional programs tend to use healthcare professionals as the sole providers. There is no study that could be identified using volunteers in transitional care programs. There were programs that used volunteers or lay persons to provide support for patients, either hospital-based or communitybased, and found them helpful to provide support to the patients. Based on findings from previous evidence which showed that transitional care model is effective and that volunteers can be conducive to patient outcomes, we built a health-social partnership transitional care model. There is a paucity of studies executing a full cost-effective analysis 
Table 1 Patient health outcomes

\begin{tabular}{|c|c|c|c|c|}
\hline & & Study group $(\mathbf{N}=\mathbf{2 7 2})$ & Control group $(\mathrm{N}=\mathbf{2 8 3})$ & P-value \\
\hline \multirow[t]{2}{*}{ Patient readmission rate $(\mathrm{N}, \%)$} & 28 days & $11,4.0 \%$ & $29,10.2 \%$ & $0.005^{\mathrm{a}}$ \\
\hline & 84 days & $22,8.1 \%$ & $55,19.4 \%$ & $<0.001^{\mathrm{a}}$ \\
\hline Readmission LOS & 28 days & $2.7(1.4,4.1)$ & $5.0(2.6,7.3)$ & $0.456^{\mathrm{b}}$ \\
\hline (Mean, 95\% Cl) & 84 days & $4.5(2.6,6.4)$ & $6.0(4.0,8.0)$ & $0.607^{b}$ \\
\hline Utility & Baseline & $0.723(0.707,0.739)$ & $0.735(0.719,0.752)$ & $0.308^{c}$ \\
\hline \multirow[t]{3}{*}{ (Mean, 95\% Cl) } & 28 days & $0.764(0.748,0.781)$ & $0.727(0.710,0.744)$ & $<0.001^{\mathrm{c}}$ \\
\hline & 84 days & $0.778(0.762,0.795)$ & $0.751(0.734,0.768)$ & $0.002^{c}$ \\
\hline & Within group comparison & $<0.001^{d}$ & $0.011^{d}$ & \\
\hline QALY gain from baseline & 28 days & $0.0016(0.0010,0.0022)$ & $-0.0003(-0.0009,0.0003)$ & $<0.001^{b}$ \\
\hline (Mean, 95\% Cl) & 84 days & $0.0089(0.0060,0.0117)$ & $0.0003(-0.0024,0.0030)$ & $<0.001^{b}$ \\
\hline
\end{tabular}

${ }^{a}$ Pearson's chi-squared test,

b Mann-Whitney U-test,

c ANCOVA test ( 28 days and 84 days adjusted by baseline),

d Repeated measures ANOVA.

on transitional care model. This study fills the knowledge gap and demonstrated that the health-social partnership transitional care program is cost effective and brings about QALY gains.

The contemporary trend in post-discharge support services advocates health and social partnership $[25,26]$ because clients returning home require different types of social support. Faulkner \& Davies [18] outlined four types of social support: instrumental support, involving the provision of tangible resources to alleviate difficulties; appraisal support, which helps individuals to evaluate the impact of situations; informational support, which provides individuals with information to deal with problems; and emotional support, which enhances self-esteem and encouragement. In this study, the nurse case managers provided all four types of support, and the volunteers backed up by the social workers provided informational and emotional support [6]. The literature has reported

Table 2 Cost of intervention and healthcare services

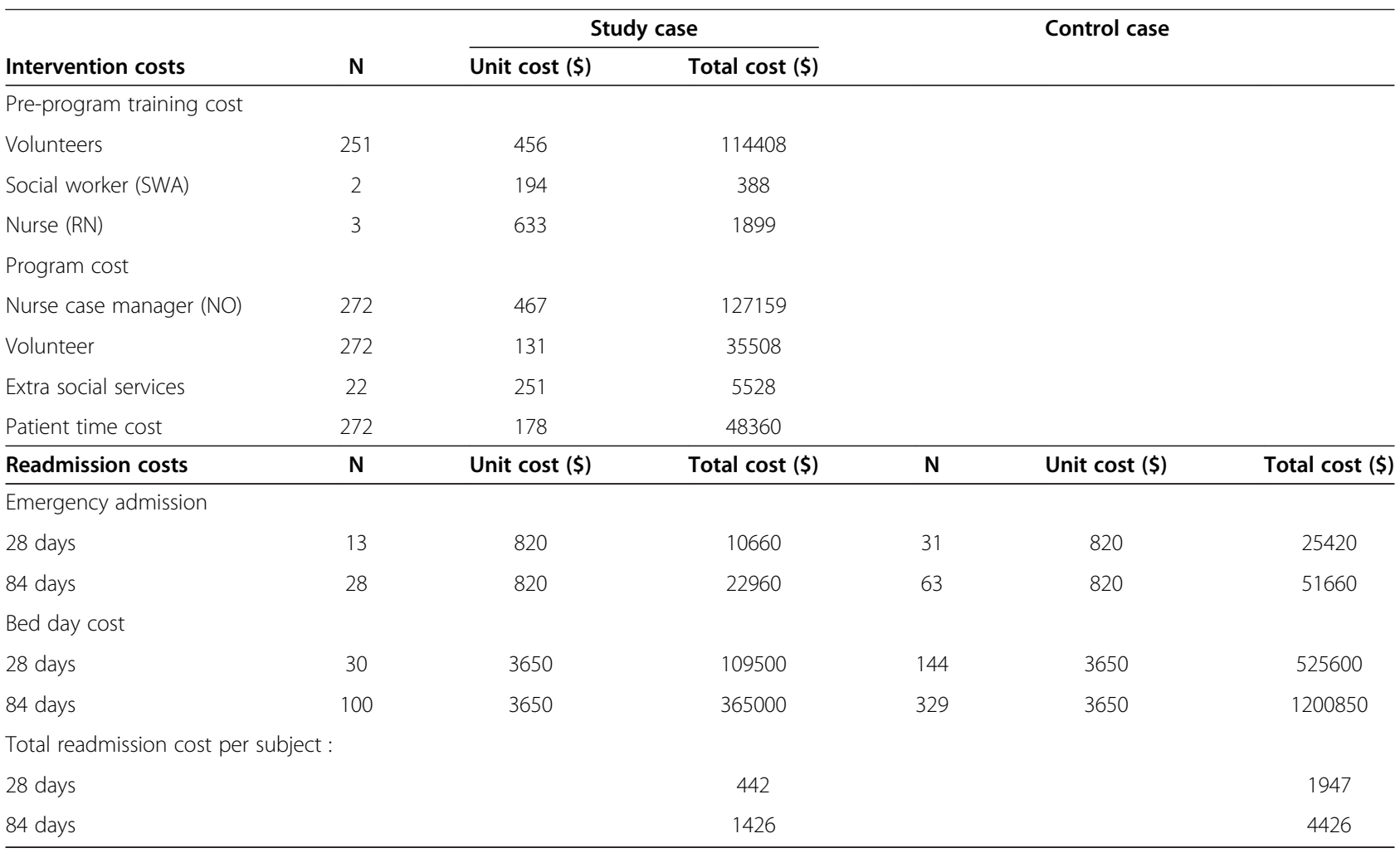


Table 3 Main results and one-way sensitivity analyses of cost-effectiveness analysis

\begin{tabular}{|c|c|c|c|c|c|c|}
\hline & \multicolumn{3}{|c|}{28 days } & \multicolumn{3}{|c|}{84 days } \\
\hline & Incremental cost (\$) & QALY gained & ICER (\$) & Incremental cost (\$) & QALY gained & ICER (\$) \\
\hline Base & -280 & 0.002 & -148828 & -1774 & 0.009 & -205079 \\
\hline \multicolumn{7}{|l|}{ Intervention cost } \\
\hline $30 \%$ lower & -648 & 0.002 & -344111 & -2142 & 0.009 & -247561 \\
\hline $30 \%$ higher & 87 & 0.002 & 46455 & -1407 & 0.009 & -162596 \\
\hline \multicolumn{7}{|l|}{ Length of stay } \\
\hline 95\% Cl minimum & 389 & 0.002 & 206455 & -910 & 0.009 & -105160 \\
\hline 95\% Cl maximum & -949 & 0.002 & -504111 & -2639 & 0.009 & -304997 \\
\hline Overall & 190 & 0.002 & 100753 & -1180 & 0.009 & -136423 \\
\hline \multicolumn{7}{|l|}{ Readmission rate } \\
\hline $30 \%$ lower & 171 & 0.002 & 91104 & -874 & 0.009 & -101072 \\
\hline $30 \%$ higher & -732 & 0.002 & -388760 & -2674 & 0.009 & -309085 \\
\hline
\end{tabular}

studies that involved volunteers in patient programs, but the findings are limited to the descriptive level. Karwalajtys et al. [27] have reported using volunteer peer educator in the community to enhance cardiovascular health awareness. Sandhaus et al. [28] have involved volunteers to help reduce delirium among elderly in the hospital. Both studies remarked that the use of volunteers
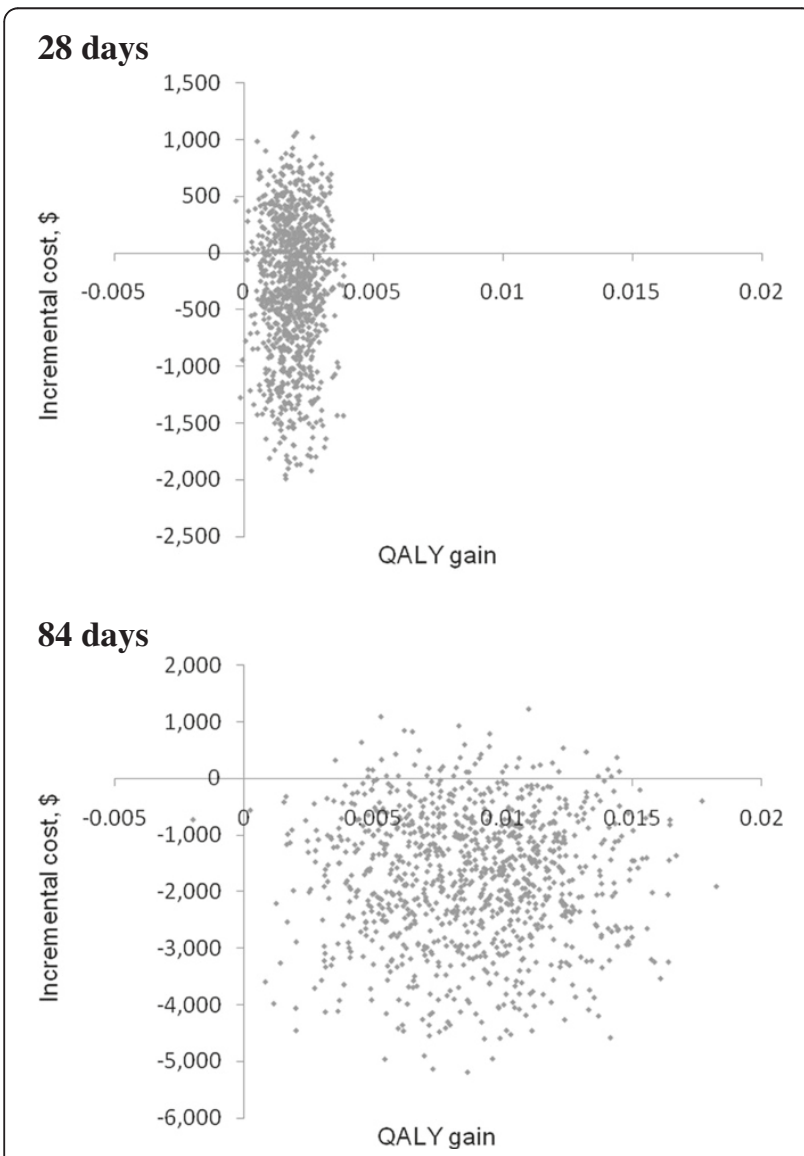

Figure 1 Cost-effectiveness plane. is a low-cost method of providing sustainable support to patients and the volunteers were welcomed by patients, their families, and nursing staff. However, no studies can be identified that report either health outcomes or costeffectiveness when volunteers are included in the patient support programs. The integration of health and social care services as a newly-developed initiative needs the support of evidence to convince policy-makers of its value in both health outcomes and cost [29]. With the demand for healthcare resources across competing programs, cost analysis in health care is essential [30].

Of all healthcare expenses, hospital use occupies the major part of the expenditure [16]. Readmission rate is a commonly used outcome in health services research, and some studies have included cost as one of their outcome variables. How do the cost-related outcomes reported in those studies compare to this study? Since the content and length of the intervention programs, as well as the type and number of providers, vary among studies, it is very difficult to make a fair comparison of the results. Also, the intervention cost varies in different

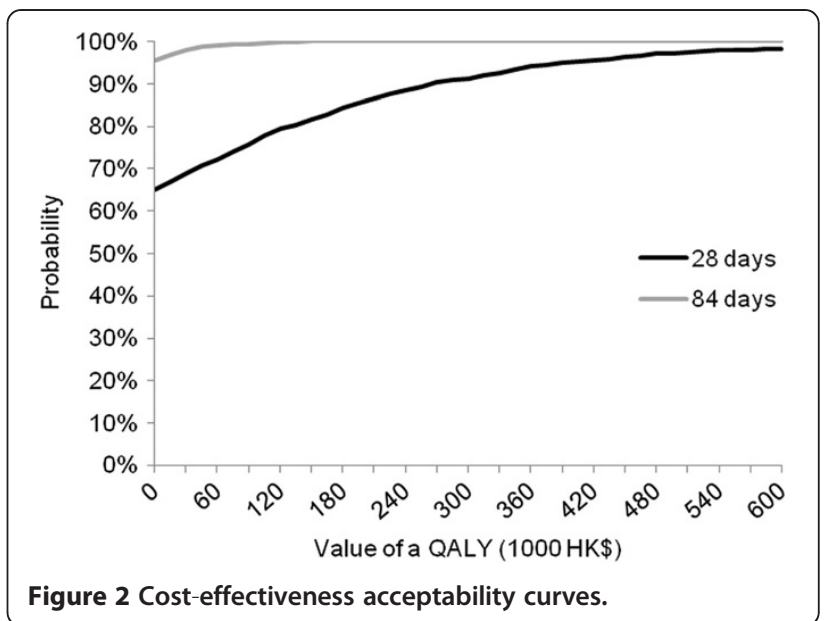


healthcare contexts. The following descriptive review will, however, help provide a synopsis of the cost savings in different places, thus helping readers to appreciate the extent of the cost savings reported in this study.

In a 12-week program with regular telephone contacts and education for heart failure patients after hospital discharge in Ireland, the cost was $€ 5860$ per patient. The intervention produced a net cost saving of $€ 37,216$ for 51 patients over 3 months [10]. Another 12-week transitional post-discharge care program introduced in the United States using advanced practice nurses also to follow up heart failure patients saved US $\$ 4,845$ per patient over a year, with the intervention and control group spending respectively US $\$ 7,636$ and US $\$ 12,481$ [4]. A 6-month program was introduced to a group of elderly patients after leaving the hospital by geriatricians targeting risk factors for preventable readmissions in France. The mean cost of the intervention was $€ 278$ per patient, and the cost savings balanced against the cost of the intervention was $€ 519$ per patient [11]. Kwok et al. [12] provided regular nurse home visits also for 6 months to discharged elderly patients in Hong Kong, and the intervention cost was US\$309 per subject. The mean total public health expenditure was reported to be lower in the intervention group, with a saving of US $\$ 2024$ per patient. For a longer program of 12 months adopted for a group of post-discharge stroke patients involving health and social care in England, the cost of domiciliary care was $£ 6840$, which was cheaper when compared to the other two modes of interventions implemented by the stroke unit $(£ 11450)$ and stroke team (£9527). The mean QALY gained were respectively $0.297,0.216$ and 0.221 for stroke unit, stroke team and domiciliary care, and there was a $59 \%$ probability of domiciliary care being cost-effective [15]. Another 1-year telephone support program was provided to community diabetes patients registered in a district in England, with the intervention estimated to cost $£ 43000 / \mathrm{QALY}$ and to have a $29 \%$ probability of being cost-effective when measured against a threshold of $£ 30000 / Q A L Y$ [31] All of the above programs used healthcare professionals as key service providers. The study by Richardson et al. [32] in England is one of the few that evaluated a program involving non-health professionals, a layperson-led self-care group. The group acted as expert patients, teaching other patients self-care support skills for long-term conditions in six weekly sessions in the community. The intervention was found to be associated with better patient outcomes at a slightly lower cost. There was a 0.020 QALY gain of the intervention group when compared with the control group, and a probability of $94 \%$ of being cost-effective when the value of $£ 20000 / Q A L Y$ was considered.

This study spent HK\$1225 (=US\$158) per patient for the study group, and the cost of readmission per subject was reduced by HK\$1490 (=US\$192) and HK\$2970 (=US\$383) respectively at 28 and 84 days. The intervention had an $89 \%$ chance of being cost-effective when checked against the NICE threshold of $£ 20000 / Q A L Y$ [23]. The expenditure figures reported in this study were substantially lower than in any of the studies reported above, and there was a high probability that the HSTCMP was effective when using the NICE threshold.

\section{Conclusions}

Studies conducted to examine the effects of a transitional care program for hospital discharged patients mainly used healthcare professionals as key providers, and the outcome measures were confined to clinical and health outcomes. This study is original in conducting a cost-effectiveness analysis that takes into account cost and outcomes of a health-social partnership transitional care model. The results supported that a program that integrates the efforts of health and social care can be cost-effective. The use of trained volunteers to support the social aspects of care helps to contain costs and produce positive outcomes. Health-social partnership is regarded as an important trend of development for postdischarge services [33]. In some countries, such as United Kingdom [34] and Sweden [25], there are national policies in place that drive and regulate health and social care integration. However, there is no reported study that helps inform the policy makers if the health-social partnership model is sound clinically and economically. This study fills the knowledge gap and adds to the literature by providing evidence to inform healthcare managers and funders that health-social transitional programs are cost-effective.

There were several limitations to this study. First, it did not include nonmedical costs such as transport costs, carer costs and the like, which may have resulted in underestimated costs from a societal perspective. Second, this study only included expenditure on hospitalization; other medical costs such as outpatient and emergency room visits, treatment, investigations, and drugs were not considered. Third, only QALY gains at 28 and 84 days after hospital discharge were examined, and there was no data available after 84 days. The sustained cost-effectiveness of this program cannot be established.

\section{Competing interests \\ The authors declare that they have no competing interests.}

\section{Authors' contributions}

FKYW designed, implemented the study and drafted the manuscript, JC, CS and SM performed the statistical analysis, drafted the results part of the manuscript, SKFT participated in the design and implementation of the study and helped to draft the manuscript. All authors read and approved the final manuscript.

\section{Acknowledgement}

We would like to thank The Hong Kong Jockey Club Charities Trust (PolyU Account 5ZH60) and the Research Grants Council of the Hong 
Kong Special Administrative Region, China (RGC Ref No 547909) to fund this study. We are indebted to the social service centers for supporting the volunteers and the volunteers who provided the social services to the clients. Our deepest thanks also go to Ms Perina Siu-Fan Li, Chief Manager, Social Service Department, The Salvation Army and Ms Janet Wai-ying Leung, Manager, Ms Sik-ying Yeung, Advanced Practice Nurse, Pre-discharge services, Queen Elizabeth Hospital for their valuable contribution in the conceptualization and implementation of the HeathSocial Partnership Transitional Program.

\section{Author details}

'School of Nursing, The Hong Kong Polytechnic University, Hunghom, Kowloon, Hong Kong, China SAR. ${ }^{2}$ School of Public Health, Department of Community Medicine, University of Hong Kong, Hong Kong, China SAR. ${ }^{3}$ Geriatric Team, Queen Elizabeth Hospital / Hong Kong Buddhist Hospital, Hong Kong, China SAR.

Received: 5 June 2012 Accepted: 19 December 2012

Published: 24 December 2012

\section{References}

1. Hammer JB: State of the Science: Posthospitalization nursing interventions in congestive heart failure. Adv Nurs Sci 2005, 28:175-190.

2. Wong FK, Mok MP, Chan T, Tsang MW: Nurse follow-up of patients with diabetes: randomized controlled trial. J Adv Nurs 2005, 50:391-402.

3. Yu DS, Thompson DR, Lee DT: Disease management programmes for older people with heart failure: crucial characteristics which improve post-discharge outcomes. Eur Heart J 2006, 27:596-612.

4. Naylor MD, Brooten DA, Campbell RL, Maislin G, McCauley KM, Schwartz IS: Transitional care of older adults hospitalized with heart failure: A randomized controlled trial. J Am Geriatr Soc 2004, 52:675-684.

5. Gonseth J, Guallar-Castillon P, Banegas JR, Rodriguez-Artalejo F: The effectiveness of disease management programmes in reducing hospital re-admission in older patients with heart failure: a systematic review and meta-analysis of published reports. Eur Heart J 2004, 25:1570-1595.

6. Wong FK, Ho MM, Yeung S, Tam SK, Chow SK: Effects of a health-social partnership transitional program on hospital readmission: A randomized controlled trial. Soc Sci Med 2011, 73:960-969.

7. Wong FK, Chow SK, Chung L, Chang K, Chan T, Lee WM, Lee R: Can home visits help reduce hospital readmissions? Randomized controlled trial. $J$ Adv Nurs 2008, 62:585-595.

8. McCusker J, Verdon J: Do geriatric interventions reduce emergency department visits? A systematic review. J Gerontol A Biol Sci Med Sci 2006, 61:53-62.

9. Courtney M, Edwards H, Chang A, Parker A, Finlayson K, Hamilton K: Fewer emergency readmissions and better quality of life for older adults at risk of hospital readmission: A randomized controlled trial to determine the effectiveness of a 24-weeks exercise and telephone follow-up program. J Am Geriatr Soc 2009, 57:395-402.

10. Ledwidge M, Barry M, Cahill J, Ryan E, Mauer B, Ryder M, Travers B, Timmons L, McDonald K: Is multidisciplinary care of heart failure costbeneficial when combined with optimal medical care. Eur $J$ Heart Fail 2003, 5:381-389.

11. Legrain S, Tubach F, Bonnet-Zamponi D, Lemaire A, Aquino JP, Paillaud E, Taillandier-Hieriche E, Thomas C, Verny M, Pasquet B, Moutet AL, Lieberherr $D$, Lacaille S: A new multimodal geriatric discharge-planning intervention to prevent emergency visits and rehospitalizations of older adults: the optimization of medication in AGEd Multicenter randomized controlled trial. J Am Geriatr Soc 2011, 59:2017-2028.

12. Kwok T, Lee J, Woo J, Lee DT, Griffith S: A randomized controlled trial of a community nurse-supported hospital discharge programme in older patients with chronic heart failure. J Clin Nurs 2008, 1:109-117.

13. Miller P, Gladman JR, Cunliffe AL, Husbands SL, Dewey ME, Harwood RH: Economic analysis of an early discharge rehabilitation service for older people. Age Ageing 2005, 34:274-280.

14. Drummond MF, Sculper MJ, Torrance GW, O'Brien BJ, Stoddart GL: Methods for the economic evaluation of health care programmes. Oxford: Oxford University Press; 2005.

15. Patel A, Knapp M, Perez I, Evans A, Kalra L: Alternative strategies for stroke care. Cost-effectiveness and cost-utility analyses from a prospective randomized controlled trial. Stroke 2004, 35:196-203.
16. Stewart S, Blue L, Walker A, Morrison C, Mcmurray JJ: An economic analysis of specialist heart failure nurse management in the UK: Can we afford not to implement it? Eur Heart J 2002, 23:1369-1378.

17. Martin K: The Omaha System: A key to practice, documentation, and information management. 2nd edition. St. Louis: Elsevier Saunders; 2005.

18. Faulkner M, Davies $\mathrm{S}$ : Social support in the healthcare setting: the role of volunteers. Health Soc Care Community 2005, 13:38-45.

19. McGhee SM, Brazier J, Lam CL, Wong LC, Chau J, Cheung A, Ho A: Qualityadjusted life years: population-specific measurement of the quality component. Hong Kong Med J 2011, 17(Suppl 6):17-21.

20. Hong Kong Census and Statistics Department Hong Kong Special Administrative Region People's Republic of China: Hong Kong Annual Digest of Statistics (2011 Edition); [http://www.statistics.gov.hk/publication/ general_stat_digest/B10100032011AN11B0100.pdf]

21. Social Welfare Department: The Government of the Hong Kong Special Administrative Region. Salary Scale of Common Posts in the Non-governmental Organisations. w.e.f 1.4.2009. [http://www.swd.gov.hk/doc/ngo/Salary\% 20Scale\%20of\%20Common\%20Posts\%20wef\%201\%204\%202009.pdf]

22. Hong Kong the Hospital Authority: Annual Plan 2011-2012; [http://www.ha. org.hk/upload/publication_12/318.pdf]

23. Carpenter J, Bithell J: Bootstrap confidence intervals: when, which, what? A practical guide for medical statisticians. Stat Med 2000, 19:1141-1164.

24. National Institute for Health and Clinical Excellence: Guide to the Methods of Technology Appraisal. 2008 [http://www.nice.org.uk/media/B52/A7/ TAMethodsGuideUpdatedJune2008.pdf]

25. Rummery K, Coleman A: Primary health and social care services in the UK: progress towards partnership? Soc Sci Med 2003, 56:1773-1782.

26. Petersson P, Springett J, Blomqvist K: Telling stories from everyday practice, an opportunity to see a bigger picture: a participatory action research project about developing discharge planning. Health Soc Care Community 2009, 17:548-556.

27. Karwalajtys T, McDonough B, Hall H, Guirguis-Younger M, Chambers LW, Kaczorowski J, Lohfeld L, Hutchison B: Development of the volunteer peer educator role in a community cardiovascular health awareness program (CHAP): A process evaluation in two communities. $J$ Community Health 2009, 34:336-345.

28. Sandhaus S, Zalon ML, Valenti D, Dzielak E, Smego RA Jr, Arzamasova U: A volunteer-based hospital elderly life program to reduce delirium. Health Care Manag 2010, 29:150-156.

29. Kodner DL: Whole-system approaches to health and social care partnerships for the frail elderly: an exploration of North American models and lessons. Health Soc Care Community 2006, 14:384-390.

30. Whitehead SJ, Ali S: Health outcomes in economic evaluation: the QALY and utilities. Br Med Bull 2010, 96:5-21.

31. Mason JM, Young RJ, New JP, Gibson JM, Long AF, Gambling T, Friede T: Economic analysis of a telemedicine intervention to improve glycemic control in patients with diabetes mellitus. Dis Management Health Outcomes 2006, 14:377-385.

32. Richardson G, Kennedy A, Reeves D, Bower P, Lee V, Middleton E, Gardner C, Rogers A: Cost effectiveness of the expert patients programme (EPP) for patients with chronic conditions. J Epidemiol Community Health 2008, 62:361-367.

33. Hudson DL: See change or quick fix? Policy on long-term conditions in England. Health Soc Care Community 2005, 13:378-385.

34. Hickey J: Integrating health and social care services. Nur Man 2008, 15:20-24.

\section{doi:10.1186/1472-6963-12-479}

Cite this article as: Wong et al:: Cost-effectiveness of a health-social partnership transitional program for post-discharge medical patients. BMC Health Services Research 2012 12:479. 